\title{
Le projet multiple des chercheurs d'aujourd'hui : entre exigence personnelle, dépendance financière et excellence formelle
}

\author{
Maxime Pauwels* \\ Écologie évolutive, Université de Lille, CNRS, UMR8198 Evo-Eco-Paleo, Lille, France
}

\begin{abstract}
Résumé - Pour les chercheurs et enseignants-chercheurs, et leurs institutions de rattachement, le concept de projet est omniprésent depuis que le Conseil européen a encouragé, en élaborant la stratégie de Lisbonne, le financement de la recherche sur projet. Il est pourtant bien rare de discuter ce que l'on entend par projet. Trois définitions non exclusives sont proposées. Le projet peut être qualifié d' «essentiel» lorsqu'il traduit l'exigence de la démarche scientifique de son porteur. Il peut être «aspirant», lorsqu'il est construit dans l'espoir d'obtenir des gratifications, souvent financières. Il peut être « coopétiteur », lorsqu'il vise à satisfaire les critères d'excellence de procédures d'évaluation. Ces définitions pourraient coexister au sein d'un projet «multiple» si elles n'étaient pas à l'origine de perspectives antagonistes générant des conflits intérieurs. Dans ce cas, il faudrait les hiérarchiser, voire en éliminer certaines, totalement ou partiellement. Les choix effectués pourraient influer sur la qualité des connaissances scientifiques produites et sur le rôle joué par ces connaissances dans les débats de société.
\end{abstract}

Mots-clés : recherche / gouvernance / méthode scientifique / financement sur projet / coopétition

\begin{abstract}
The multiple project of today's scientists: maneuvering between personal requirements, financial dependence, and formal excellence. The concept of "project", that usually implicitly refers to "scientific project", has spread invasively in the daily life of academic scientific research. This noticeably started in 2000, after the European Council promoted through the Lisbon strategy the allocation of research funds on a competitive basis, for example opening calls for project proposals and providing financial support to top-ranked ones only. Nevertheless, project proponents rarely question what a project actually is. Three non-exclusive definitions of the concept are proposed in this paper. First, a scientific project can be "essential", when it agrees with the way scientists consider how science works in theory and satisfies personal methodological requirements. Second, a scientific project can be "aspirant", when it is specifically formatted to fit project selection criteria, those of open calls and funding programs for example, and in order to outcompete rival proposals. Third, a scientific project can be "coopetitive", when it is a mean to boost personal carreer in an evaluation context mostly based on quantitative scientific production. Theoretically, it can be expected that today's scientists manage a "multiple" project that would combine various objectives corresponding to these types of project. However, the coexistence of potentially antagonist objectives (e.g. quality versus quantity) could raise internal conflicts. Then, regaining personal balance might require to selectively drop some objectives. Depending on those that are maintained, this could impact the quality of future science work as well as the role that science could play in societal debates.
\end{abstract}

Keywords: research policy / science method / project funding / evaluation / coopetition

Le concept de projet est actuellement l'un des buzzwords (Bensaude-Vincent, 2013) les plus camouflés de la recherche académique. Non content d'absorber régulièrement l'attention de ses acteurs, il est l'une de

\footnotetext{
*Auteur correspondant : maxime.pauwels@univ-lille1.fr
}

leurs plus profondes obsessions. Chercheurs et enseignantschercheurs, mais aussi, en amont d'eux, tous ceux qui les administrent et, en aval d'eux, les étudiants qui aspirent à intégrer le monde actif de la science, tous, de façon récurrente, volontairement ou sous la contrainte, définissent, redéfinissent, écrivent, réécrivent, soumettent, 
resoumettent, coordonnent, administrent, valorisent, etc., leurs projets. Pour autant, sont-ce des projets scientifiques? Et d'ailleurs, qu'est-ce qu'un projet scientifique?

Voilà une question paradoxalement peu discutée... par les scientifiques eux-mêmes. Car il en va autrement aux frontières de leur communauté. En effet, depuis le Conseil européen de mars 2000 qui a conduit à l'élaboration de la stratégie de Lisbonne et à la réflexion concomitante sur l'économie de la connaissance, de nouvelles conceptions du projet scientifique, associées au développement de la recherche sur contrat, nourries d'intentions politiques, rivalisent avec d'autres conceptions, plus traditionnelles, moins délibérées.

Proposer de nouvelles conceptions, c'est potentiellement modifier le quotidien de la science. Car la définition du concept de projet scientifique peut être performative dans sa capacité à déterminer les modalités d'exercice de la science. En effet, redéfinir ce qu'est un projet scientifique, n'est-ce pas aussi reconsidérer pourquoi, comment, on mène ses activités scientifiques? Motivé par cette question, le présent article propose de discerner trois conceptions du projet (du) scientifique, non exclusives mais répondant à des objectifs distincts, en essayant d'y associer un comportement. Il conclut sur la possibilité de coexistence de ces trois conceptions et sur les conséquences possibles de leurs interférences sur le devenir de la recherche scientifique.

\section{Le projet scientifique essentiel}

Une compréhension commune du concept de projet scientifique essentiel pourrait rappeler pour l'illustrer les ambitions d'illustres scientifiques qui ont marqué l'histoire occidentale. Les objectifs copernicien, darwinien ou freudien de comprendre, par l'analyse critique des observations et/ou résultats d'expérience, la place de l'homme dans l'Univers, le monde vivant et son évolution, ou la maîtrise de soi étaient sans aucun doute des projets scientifiques. Ces projets, comme d'autres, moins emblématiques mais menés avec une discipline comparable, consubstantiels aux existences de leurs porteurs, les ont animés d'une volonté que d'aucuns n'hésiteraient pas à considérer comme alimentée par une croyance, sinon religieuse, au moins métaphysique, dans la primauté de la démarche scientifique. Celle qui refoule les a priori de toutes sortes derrière une exigence méthodologique, faite de doute constructif, d'observations matérialistes et de rationalité rigoureuse (Lecointre, 2018). Cela jusqu'à valoir parfois à ces savants la résistance de la majorité (visible) de leurs contemporains, défenseurs de visions plus valorisantes, plus confortables, plus faciles, pour eux-mêmes du moins, $\mathrm{du}$ monde. À l'extrême, des projets scientifiques ont pu conditionner et déterminer, parfois tragiquement, l'existence des scientifiques qui les incarnaient.
Cette volonté anime toujours aujourd'hui les étudiants, chercheurs et enseignants-chercheurs qui considèrent que la connaissance n'a de valeurs que lorsqu'elle est évolutive, issue d'une démarche exigeante qui, loin de craindre la réalité des faits observables, s'y confronte pour mieux les intégrer. C'est d'ailleurs encore accompagnés de cette volonté que l'on présente parfois les scientifiques aux enfants, pour leur donner le goût des sciences, ou qu'on les représente dans les fictions littéraires ou cinématographiques (Stengers, 2013).

Dans l'idéal, cette volonté s'accompagne de la défense d'une science libre, indépendante, apolitique. Pour autant, bien que possiblement pénétrée de fantasme, elle ne réduit en rien le scientifique au scientiste. D'une part, s'imposer la rigueur intellectuelle associée à la démarche scientifique et, d'autre part, considérer que les résultats scientifiques participent seuls à l'actuelle compréhension du monde, qu'ils pourvoiront immanquablement des progrès techniques requis pour le délivrer de toute limite, toute menace, durable, ou qu'ils guideront en maitres les choix politiques futurs, sont des positions dissociables. Tout scientifique du XXI ${ }^{\mathrm{e}}$ siècle a pu prendre acte, à la lecture de l'histoire récente, des insuffisances et détournements potentiels des avancées scientifiques, et il comprend que si la science est un idéal, sa pratique n'est qu'un effort vers cet idéal. En conséquence, être essentiellement scientifique, malgré la prétention inhérente aux ambitions associées, c'est désormais aussi comprendre que si la science accumule avec méthode les connaissances qui pourront orienter les choix politiques, ses limites lui interdisent d'effectuer ces choix. C'est admettre, plus humblement, que la connaissance scientifique évoluera plus paisiblement en interactions, dans la discussion plutôt que dans la confrontation, avec d'autres formes d'appréhension du monde.

\section{Le projet scientifique aspirant}

À tous les niveaux de l'organisation actuelle de la recherche scientifique, un autre concept de projet scientifique montre une vitalité envahissante. Il se répand, comme un leitmotiv, avec une périodicité circadienne, lorsqu'il s'agit, au quotidien, de gérer administrativement ses projets; avec une périodicité annuelle, lorsqu'il s'agit, à dates quasi-fixes au fil des saisons, d'épier, de compulser, de s'adapter et de répondre aux multiples «appels à projets » (AAP); avec une périodicité pluriannuelle, lorsqu'il s'agit, lors de candidatures ou d'évaluations, d'exposer ses projets actuels et futurs, associant, à l'évidence, qualité, faisabilité et applicabilité. Ce concept-là n'a pas que les chercheurs pour hôtes, mais aussi leurs laboratoires, leurs établissements, voire leurs gouvernements. C'est celui du projet scientifique aspirant, notamment à être financé, sur contrat. 
En mars 2000, à Lisbonne, parmi les multiples propositions avancées lors du Conseil européen pour faire de l'Union européenne "l'économie de la connaissance la plus compétitive et la plus dynamique du monde»(Conseil européen, 2000), il est proposé d'encourager le financement de projets sur contrat plutôt qu'à travers des budgets récurrents. Cela se traduit notamment en 2007 par l'institution du Conseil européen de la recherche (acronyme «CER» inconnu, car inusité, étouffé par sa version anglaise «ERC» pour European Research Council) qui «a pour mission d'encourager la recherche de la plus haute qualité en Europe grâce à un financement concurrentiel ${ }^{1}$ "), c'est-à-dire via des AAP auxquels répondent les chercheurs. «L'objectif est d'identifier les meilleures idées, et de retenir les "meilleurs cerveaux" en Europe", et de leur accorder des bourses sur plusieurs années. L' " excellence scientifique », évaluée par des pairs, est présentée comme le seul critère de sélection, même si on lit parfois que l'innovation ("groundbreaking nature») et l'ambition sont aussi souhaitables.

En France, depuis 2005, ce nouveau mode de pilotage de la recherche se traduit, notamment, par la création de l'Agence nationale de la recherche (ANR) qui a pour mission «[la mise] en œuvre du financement de la recherche sur projets ${ }^{2} »$ en France et qui ouvre régulièrement des AAP auxquels peuvent candidater, sous leur nom et rattachés à une institution, des chercheurs intronisés «coordinateurs», pour demander le financement de leur projet aspirant, seuls ou en collaboration avec des «partenaires » selon les formules. Conséquence: il n'y a plus que peu, s'il en reste, de projets scientifiques d'envergure qui puissent être menés par leurs porteurs sans qu'ils soient aspirants, et donc supportés par un ou des organismes financeurs. Corollaire: les activités de recherche ne sont plus que marginalement soutenues par des budgets récurrents, subsidiaires.

Suit, en 2007, l'autonomisation des universités, c'està-dire la possibilité qui leur est donnée de gérer « ellesmêmes leurs ressources humaines et la masse salariale qui va avec», "par une loi qui a changé le visage des universités et la compétitivité des territoires » (MESRI, 2011). Elles deviennent alors susceptibles de répondre à des AAP. Aujourd'hui, les universités, comme leurs laboratoires, comptent donc sur le succès de leurs candidatures à divers AAP, et sur l'obtention de contrats avec les organismes, publics ou privés qui les lancent, pour couvrir financièrement leurs projets. C'est le cas par exemple dans le cadre du Programme d'investissements d'avenir (PIA), initié en 2010 par l'État français (MESRI, 2012), qui proposait dans sa première phase

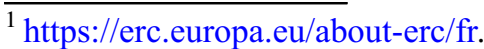

${ }^{2}$ https://anr.fr/fr/lanr-et-la-recherche/missions.
}

(PIA1, 2010-2012) de distinguer, pour les soutenir financièrement, des projets aspirants soumis par les laboratoires et universités. Les lauréats sont aussi gratifiés de labels, évidemment d' «excellence» : chaire d'excellence, laboratoire, équipement, initiative d'excellence (respectivement Labex, Équipex, Idex). Même si le vocabulaire évolue, la logique comme les objectifs sont maintenus dans les phases 2 (PIA2, 2013-2016) et 3 (PIA3, 2017-) : « encourager la recherche et l'innovation et faire basculer la France dans la société de la connaissance », "retrouver le chemin d'une croissance forte et durable».

Multiples sont les voix chagrines, individuelles ou collectives, qui ont pu s'élever pour regretter le coût, en unités de temps consacré, et donc économique, que peuvent représenter, antérieurement, l'élaboration d'une candidature et, postérieurement, pour les lauréats, les charges administratives consécutives à son succès. Tout aussi audibles sont celles qui soulignent la nécessaire mise en compétition que génèrent les offres proposées, ou le discriminant procédé qui implique par principe l'élection de quelques-uns et l'éviction de tous les autres. Plus sourdes semblent être celles qui s'interrogent sur les conséquences plus profondes sur les modes d'élaboration des projets de recherche.

Les AAP de recherche sont pour la plupart lancés avec une liste d'axes prioritaires dans lesquels les projets candidats devront se placer s'ils veulent espérer être lauréats. En parallèle de ses AAP, l'ANR publie ainsi un plan d'action ${ }^{3}$ déclinant jusqu'à l'abstrus ${ }^{4}$ des dizaines d'axes de recherche thématiques prioritaires, parmi lesquels les projets aspirants doivent s'insérer. Ces axes, définis politiquement, doivent aussi circonscrire une recherche favorable au redressement (économique) de la France. Un tel contrôle des thématiques de recherche supportées représente l'avènement du «capitalisme cognitif» ou de l'«économie de la connaissance», promus par l'Union européenne dans la déclaration de Lisbonne, qui considère que la production de savoir est un pilier du développement économique... et doit donc suivre des orientations politiquement déterminées. Le succès aux AAP est soumis aussi parfois à des contraintes structurelles: ainsi le regroupement, voire la «fusion », des universités à l'échelle régionale est une condition sine qua non pour accéder au podium des Idex, malgré les conséquences constatées - inévitables quand la motivation est extrinsèque ? - sur les conditions de travail (Gérand, 2016).

\footnotetext{
${ }^{3}$ https://anr.fr/fileadmin/aap/2020/aapg-2020-v1.4.pdf.

${ }^{4}$ Pour comprendre le risque de glissement vers l'absurde lié à la définition déconnectée des plans d'action, parcourir le site satirique de l'ANES (Agence nationale de l'excellence scientifique): http://excellagence.fr.
} 
Outre qu'il n'appartient plus aux chercheurs de définir leur projet de recherche, cette dépendance politique contraint les lauréats - et a fortiori, l'essaim jusqu'à dix fois plus considérable des projets soumis - à une convergence vers les préoccupations politiques de l'époque. Par exemple, aujourd'hui, quelle que soit la discipline concernée, dans un contexte où les États de l'Union européenne s'engagent officiellement à financer la lutte contre le changement climatique, difficile de ne pas associer aux propositions de projets le mot-clé «climate change » (in English please). Au prix parfois d'un effort de contorsionniste, voire d'avilissement ou de prostitution (Stengers, 2013), pour se conformer aux attentes des financeurs, quand cela n'aboutit pas tout simplement à une réorientation substantielle des thématiques de recherches, nonobstant l'expérience accumulée par les aspirants dans leurs travaux précédents, sur d'autres voies.

Enfin, si le financement sur projet entraine une canalisation des thématiques de recherches qui peuvent être abordées, il aboutit aussi à une standardisation des projets élaborés. Sur la durée tout d'abord, car un projet aspirant bien construit doit annoncer le temps nécessaire à sa réalisation. Durée nécessairement courte, car les financements obtenus ne sont disponibles le plus souvent que pour quelques années, au-delà desquelles ils seront perdus. Sur le niveau d'audace, ensuite. Car le projet aspirant doit annoncer les résultats qui sont attendus, dans quels délais et donc dans quel ordre (cette prescience obligée interroge sur le succès médiatique récent du concept antagoniste de sérendipité [Catellin, 2014], qui célèbre l'inattendu en science). Plutôt que des résultats, les projets aspirants annoncent, dans un vocabulaire formaté, des «délivrables», usant ainsi de l'anglicisme d'un adjectif promu nom commun, qui implique quasi subliminalement que ces résultatsdélivrables seront rendus disponibles (soit!) pour pouvoir être valorisés et participer au développement économique.

\section{Le projet scientifique coopétiteur}

Le développement de la recherche sur projet n'est que la dimension formelle du processus de marchandisation de la science. En parallèle, mais non sans relation, car c'est devenu aussi un élément majeur dans l'appréciation des projets aspirants soumis aux AAP, on a pu voir, dans les deux décennies précédentes, se développer, comme viralement, des outils nouveaux d'évaluation des chercheurs et de leurs laboratoires ou institutions.

Les outils d'évaluation sont multiples, mais leurs logiques reposent fortement sur un nombre limité d'indicateurs, pour la plupart bibliométriques. Ces indicateurs sont calculés à partir de données relatives aux publications scientifiques, en se focalisant sur les articles des journaux à comité de lecture, et en excluant la littérature grise ou toute forme de publication adressée au grand public.

Le plus brut de ces indicateurs est le facteur d'impact (FI) de la revue dans laquelle sont publiés les travaux. Pour les journaux qui peuvent se targuer d'en posséder un, le FI est calculé comme la moyenne des citations des articles publiés au cours des deux années précédentes. Développé à l'origine pour quantifier la réputation d'une revue, il est aujourd'hui utilisé, non sans critique (Gingras, 2014), pour évaluer qualitativement la production des chercheurs.

Derrière ce dévoiement s'embusquent trois apories. La première est que le publiant n'est pas le publieur: comment un outil développé pour classer des journaux permet-il d'apprécier ses contributeurs? La seconde est qu'une moyenne n'informe pas sur la distribution des contributions particulières des différents auteurs au succès du journal: dans un journal à fort FI, une majorité d'articles peut n'être que peu citée. La troisième est que la quantité n'est pas la qualité : un article peut être très cité parce que très critiquable, ou un autre peu cité parce que novateur et mal compris. Un autre indicateur, plus proche de l'évalué, est son nombre de publications, mais il ne tient compte ni de sa contribution à la publication lorsque la liste des auteurs s'allonge - jusqu'à plusieurs centaines parfois -, ni (ici non plus) de la qualité du contenu des publications. Certains indicateurs bibliométriques se veulent plus synthétiques, comme l'indice de Hirsch (ou indice h), qui est égal pour un chercheur au nombre $\mathrm{N}$ de ses articles qui ont reçu $\mathrm{N}$ citations ou plus. Enfin, d'autres indicateurs semblent s'inspirer de la bibliométrie pour produire à travers un calcul savant, mais sibyllin, un score qui permet à l'évalué de se classer parmi ses pairs. C'est le cas du RG Score produit par le réseau social ResearchGate, le «Facebook des scientifiques», spams compris (Fagot, 2017). Ici encore, le calcul de l'indicateur ne nécessite pas de s'intéresser au contenu des publications.

On peut comprendre cette exploitation de la bibliométrie par les tenants d'une gestion managériale de la recherche scientifique, qui considèrent que la motivation, donc l'investissement et surtout in fine la productivité du chercheur, se dope par l'injection facilitée et régulière d'indices de performances individuels, stimulant la poursuite d'un palmarès supérieur à ceux de ses semblables (Bruno, 2008). Car l'évaluation s'accompagne en effet toujours de comparaisons, permises par le positionnement des «scores» obtenus par les évalués dans la distribution des valeurs obtenues par leurs pairs aux mêmes indices. On s'étonne encore, pourtant, connaissant le manque de rigueur de cette méthodologie, que nombre des chercheurs, à qui on aime attribuer une forte exigence intellectuelle, se soumettent à la primauté des indicateurs bibliométriques dans 
l'évaluation. En effet, publier dans un journal à haut FI devient pour beaucoup un objectif majeur. Accumuler les publications, quitte à les multiplier à l'excès à partir d'une même étude, au risque de partitionner artificiellement les données acquises, devient une stratégie efficace.

Cette réorientation des objectifs de carrière vers la performance quantitative pourrait rester anodine si elle s'effectuait sans ingénuité, voire avec cynisme, consciente qu'il ne s'agit que d'une conformation superficielle aux demandes des instances évaluatrices. Mais le doute est permis. Car ce sont les chercheurs et enseignants-chercheurs eux-mêmes qui bien souvent évaluent leurs pairs. Que ce soit formellement, en participant, par exemple, aux sessions du Conseil national des universités $\left(\mathrm{CNU}^{5}\right)$, aux comités de sélection lors de concours de recrutement, aux comités d'évaluation du Haut Conseil de l'évaluation de la recherche et de l'enseignement supérieur $\left(\right.$ Hceres $\left.^{6}\right)$, ou informellement, dans les discussions de couloirs, au laboratoire, en congrès, à la pause-café. Et le jugement sur les pairs est souvent d'abord orienté par le bilan bibliométrique demandé dans leur curriculum vitæ. Publier "beaucoup» dans les «gros» journaux est le superpouvoir des héros modernes de la recherche scientifique. Et c'est presque inconsciemment que l'autoévaluation suit la même logique, jusqu'à la manie, tant l'imprégnation peut être forte dans un environnement qui semble suivre unanimement une même idéologie. Publier de nouveaux articles ne devient alors plus tant le moyen de faire connaître ses nouvelles découvertes qu'une forme d'accumulation de capitaux, transformant la valorisation de la recherche scientifique en source d'enrichissement personnel.

(Se) donner pour horizon des bornes bibliométriques n'est, finalement, pas sans conséquence sur la pratique de la recherche. Car pour bien publier, il faut publier neuf et vite. Publier neuf, idéalement sur des sujets à fort potentiel médiatique, c'est publier sur un sujet sur lequel personne n'a jamais travaillé, dans une relative discrétion pour que personne ne l'apprenne et ne s'y investisse. Publier vite, c'est s'intéresser à un sujet rentabilisable à court terme. Dans l'éventualité où d'autres mèneraient des recherches sur le même sujet, c'est aussi être le premier à publier sur une thématique, au risque sinon de voir l'intérêt des journaux chuter, ainsi, conséquemment, que le FI des revues qui restent susceptibles d'accueillir son article. Le climat est donc désormais à la compétition, car lorsque l'on veut être le meilleur, l'autre devient nécessairement un concurrent.

\footnotetext{
${ }^{5}$ www.conseil-national-des-universites.fr.

${ }^{6}$ www.hceres.fr.
}

Entrer dans la compétition lorsque l'on souhaite parallèlement construire des projets à soumettre aux AAP, qui se doivent d'être collaboratifs, peut paraître paradoxal. Car comment peut-on à la fois vouloir distancer ses pairs et faire équipe avec eux? En étant simplement logique et cohérent; en évaluant ses pairs, en les sélectionnant avant d'en faire ses collaborateurs; en entrant dans la coopétition: la collaboration comme moyen pour la compétition. Le développement d'un projet individuel coopétiteur est l'objectif ultime des outils développés. C'est le principe dominant dans la mêlée actuelle de scientifiques-gladiateurs jetés dans l'arène par quelques décideurs, sur les conseils des quelques théoriciens du management, et dont seuls quelques-uns, les excellents, sortiront, saufs mais matés, enduits d'un succès maintenant dans l'ombre la faillite de tous les autres.

\section{Vers un projet multiple en tension}

Les trois concepts de projet évoqués (essentiel, aspirant, coopétiteur), en dépit de leurs différences, ne sont pas nécessairement exclusifs. Des interactions sont même facilement concevables. Un projet essentiel motiverait, par exemple, la construction d'un projet aspirant qui, réciproquement, s'il était lauréat, permettrait, à court ou moyen terme, la mise en œuvre concrète $\mathrm{du}$ projet essentiel. On prétend aussi que l'effort de construction du projet aspirant imposerait une réflexion bénéfique à la conduite du projet essentiel. C'est ainsi que, dans cet esprit, l'ERC assure ne pas vouloir orienter les thématiques de recherches à travers ses AAP. Par ailleurs, le projet aspirant (lauréat) pourrait alimenter un projet coopétiteur, qui requiert la construction d'un palmarès incluant les succès aux AAP. L'ERC, toujours, est, par exemple, soucieux de mesurer l'impact de ses bourses sur l'évolution des carrières. Enfin, dans la mesure où, à tort ou à raison, les grandes avancées scientifiques sont presque toujours associées à une personne, parfois deux, plus rarement trois, le projet coopétiteur pourrait, dans certaines conditions, fournir la source d'énergie requise pour atteindre les niveaux de rigueur et d'endurance nécessaires à la réalisation d'un projet essentiel. On pourrait donc s'attendre aujourd'hui à voir cohabiter chez un même chercheur les différents types de projets.

Il s'agirait alors pour lui de devoir gérer intimement un projet global que l'on peut dire «multiple», comme on parle de personnalité multiple. Cela impliquerait de suivre, sur le long terme de la carrière professionnelle, voire du parcours personnel - car la lisière entre les deux est souvent perméable-, une trajectoire finalement unique mais qui puisse englober et articuler, avec des dynamiques variables, des aspirations multiples, idiosyncrasiques ou circonstancielles, assumées ou subies, 
en fonction du type de projet auxquelles elles correspondent. Ces aspirations consubstantielles seraient plus prosaïques, vénales et égotiques, lorsqu'il faut, par exemple, comme le contexte structurel demande à le faire, convaincre financeurs et autres évaluateurs de la valeur de ses projets et de soi-même; elles seraient plus ambitieuses, transcendantes et altruistes, lorsqu'il faut, par exemple, comme une adhésion profonde et désintéressée à la méthode scientifique le motiverait, convaincre de la valeur de la connaissance scientifique pour le devenir de nos sociétés.

Cependant, il est peu probable que les trois types de projets coexistent également, ni même équitablement, à hauteur des investissements qu'ils exigent respectivement, dans la conscience, sinon l'inconscience, du chercheur. D'abord, parce que nul n'est capable d'assumer des tâches nouvelles, comme celles associées dernièrement aux projets aspirants et coopétiteurs, à l'infini, sans réduire le volume des efforts consentis auparavant à celles qui préexistaient. Il faudrait s'attendre alors à des choix. Ensuite, parce que la situation est déséquilibrée: les encouragements à l'excellence par les autorités portent essentiellement sur une réussite envisagée quantitativement en termes d'articles publiés et de projets aspirants financés, plutôt que sur la qualité des résultats scientifiques obtenus, dont on discute finalement assez peu la portée. Il faudrait s'attendre alors naturellement à l'émergence des stratégies opportunistes priorisant ce qui, dans ces circonstances, est rapidement valorisant. Enfin, et c'est peut-être le plus insurmontable, sans même devoir classer moralement les objectifs liés aux trois types de projets, il est à craindre qu'ils soient si hétérogènes que les unir dans un même être puisse être à l'origine de troubles, voire de conflits, intérieurs. Ceux-ci ont d'ailleurs déjà été évoqués dans divers documents qui, soit rétrospectivement, témoignent de la dégradation des conditions de travail dans les laboratoires à la suite des évolutions récentes du pilotage de la recherche (COMETS, 2014a; COMETS, 2014b; Jacq, 2016), soit par anticipation, proposent de guider étudiants et chercheurs afin qu'ils sachent se satisfaire des conditions actuelles d'exercice de la recherche scientifique (Snieder et Schneider, 2016). Il faudrait s'attendre, alors, aux développements de mécanismes de résistance psychologique, amenant à l'exclusion de certains objectifs, pour retrouver plus d'homogénéité et de sérénité.

\section{Conclusion}

L'ambition fondamentale de la science est de produire des connaissances universelles, dont la construction ne doit pas dépendre de la culture ou des opinions personnelles des contributeurs. L'objectivation des savoirs s'effectue notamment à trois conditions : l'indépendance de la démarche scientifique, la validation des résultats et de leurs interprétations par des collectifs de scientifiques plutôt que par des individus, la confirmation des résultats par la répétition des expériences menées pour les obtenir (Lecointre, 2018). Le poids récemment augmenté des considérations politiques dans l'orientation des projets de recherche, la dépendance financière, les conséquences néfastes de la compétition, comme de la coopétition, sur la capacité des chercheurs à contribuer aux travaux de leurs pairs ou à les juger librement, ainsi que la quête de rapidité et de nouveauté dans l'acquisition, voire l'accumulation, de résultats, nuisent à ces trois conditions. Le risque est de voir se développer une science domestiquée, à l'évolution contrôlée par des considérations politiques et aux résultats devant être exploitables sur le court terme, ou une mauvaise science aux travaux certes publiés sur des critères bibliométriques, mais au détriment de la rigueur et de la prise de distance qui devraient les accompagner (Smaldino et McElreath, 2016). Cela ne peut que nuire au rôle du scientifique dans les nombreux débats de société actuels, qui requièrent une culture scientifique pour être correctement menés. Dans un monde complexe, dont la direction irénique nécessite de pouvoir s'appuyer sur des connaissances objectives, en tout cas acquises sans autre objectif qu'elles-mêmes, sans conflit d'intérêts, cette évolution est à regretter.

\section{Références}

Bensaude-Vincent B. 2013, Le bourdonnement des technosciences. Réflexions sur quelques buzzwords, Alliage, 72, 23-29, http://revel.unice.fr/alliage/index.html?id=4153.

Bruno I., 2008. À vos marques ${ }^{\circledR}$, prêts.. cherchez! La stratégie européenne de Lisbonne, vers un marché de la recherche, Paris, Éditions du Croquant.

Catellin S., 2014. Sérendipité. Du conte au concept, Paris, Le Seuil.

COMETS (Comité d'éthique du CNRS) , 2014a. Problèmes éthiques pour les métiers de la recherche publique en mutation. Autosaisine, février, COMETS, CNRS, www. cnrs.fr/comets/spip.php?article95.

COMETS (Comité d'éthique du CNRS), 2014b. La politique de l'excellence en recherche. Co-saisine de la présidence $d u$ CNRS et du Comité d'éthique, mai, www.cnrs.fr/comets/ spip.php?article99.

Conseil européen, 2000. Conclusions de la présidence, sommet de Lisbonne, 23-24 mars, Nr:100/1/00, § 5, www.europarl. europa.eu/summits/lis1_fr.htm.

Fagot V., 2017. ResearchGate, le succès discret du «Facebook des scientifiques », Le Monde, 4 mars, www.lemonde.fr/ pixels/article/2017/03/04/researchgate-le-succes-discret-dufacebook-des-scientifiques_5089202_4408996.html.

Gérand C., 2016. Aix-Marseille, laboratoire de la fusion des universités, Le Monde diplomatique, 750, 21-22, www. monde-diplomatique.fr/2016/09/GERAND/56207.

Gingras Y., 2014. Les dérives de l'évaluation de la recherche. Du bon usage de la bibliométrie, Paris, Raison d'Agir. 
Jacq A., 2016. Les nouveaux modes de pilotage de la recherche: Quels effets ? Le séminaire 2014-2015 du centre d'Alembert de l'Université Paris-Sud, Natures Sciences Sociétés. 24, 4, 371-378, DOI : 10.1051/nss/ 2017002.

Lecointre G., 2018 [1 ${ }^{\text {re }}$ éd. 2012]. Les sciences face aux créationnismes. Ré-expliciter le contrat méthodologique des chercheurs, Versailles, Quæ.

MESRI (ministère de l'Enseignement supérieur, de la Recherche et de l'Innovation), 2011. Guide pratique de l'autonomie des universités, www.enseignementsup-recher che.gouv.fr/pid24841/guide-pratique-autonomie-des-uni versites.html.
MESRI (ministère de l'Enseignement supérieur, de la Recherche et de l'Innovation), 2012. Comprendre le Programme d'investissements d'avenir, www.enseigne mentsup-recherche.gouv.fr/cid55892/comprendre-le-pro gramme-investissements-d-avenir.html.

Smaldino P.E., McElreath R., 2016. The natural selection of bad science. Royal Society Open Science, 3, 160384, DOI: 10.1098/rsos.160384.

Snieder R., Schneider J., 2016. The joy of science. Seven principles for scientists seeking happiness, harmony, and success, Cambridge, Cambridge University Press.

Stengers I., 2013. Une autre science est possible !, Paris, La Découverte.

Citation de l'article : Pauwels M., 2019. Le projet multiple des chercheurs d'aujourd'hui: entre exigence personnelle, dépendance financière et excellence formelle. Nat. Sci. Soc. 27, 3, 350-356. 